\title{
Structure parameter correlation of some quinoxaline derivatives through IR and ${ }^{13} \mathrm{C}$ NMR spectra
}

\author{
G. Thirunarayanan ${ }^{1, *}$, I. Muthuvel ${ }^{1}$, V. Sathiyendiran ${ }^{2}$ \\ ${ }^{1}$ Department of Chemistry, Annamalai University, Annamalainagar - 608 002, India \\ ${ }^{2}$ Department of Chemistry, Sourashtra College, Madurai - 625 004, India \\ *E-mail address: drgtnarayanan@gmail.com
}

\begin{abstract}
A series of 6-substituted quanoxaline derivatives have been synthesized and examined their purities by literature method. The infrared and ${ }^{13} \mathrm{C}$ NMR spectral data of these quinoxalines were correlated with Hammett substituent constants, $\mathrm{F}$ and $\mathrm{R}$ parameters using single and multi-regression analysis. From the results of statistical analysis, the effect of substituents on the spectral frequencies has been studied.
\end{abstract}

Keywords: Quinoxalines; IR spectra; NMR spectra; Hammett Correlation; Substituent effects

\section{INTRODUCTION}

Quinoxaline derivatives are medicinally important bi nitrogen heterocycles due to presence of this ring in numerous antibiotics [1,2]. The important biological activities of quinoxalines are antibacterial [2,3], antiviral [4], anti-inflammatory [4], askinase inhibitors [4], anticancer [4], antimycobacterial [5,6], antifungal [7], anthelmintic [4,7], antidepressants [8] and antitumour [9,10]. Many kinds of synthetic methods including solvent-free and catalysts were reported in the literature for synthesis of quinoxalines by the condensation of 1,2-diamines and 1,2-dicarbonyl compounds [1,2,11].

Spectroscopic data have been employed for studying the molecular equilibration of carbonyl compounds, pyrazolines and imines [12-14]. The E- and Z-notation of alkenes including $\alpha, \beta$-unsaturated carbonyl compounds and the stereochemistry of protons in the pyrazoline derivatives are confirmed by IR and ${ }^{1} \mathrm{H}$ NMR spectroscopy [12-14]. The qsar and qspr study was established with various compounds such as chalcones, [12] pyrazolines [13], imines [14], flavones [15], pyrimidines [16], carboxamides [17], oxazines [18], epoxides [19], sulfonamides [20], acyl bromides [21], Tröger's bases [22], di-imines [23] and thiadiazoles [24]. Recently Thirunarayanan and Sekar was studied the spectral qsar correlations in the pyrazoline derivatives [25]. The correlation study of infrared and ${ }^{13} \mathrm{C}$ NMR chemical shifts of aryl hydrazides with Hammett substituent constants and F and R parameters were established by Thirunarayanan et al., [26] Mayavel et al have studied the spectral correlations of infrared and NMR spectral frequencies of carbazole imines with Hammett substituent constants, F and R parameters [27]. Sathiyendiran et al. [28] have prepared some 2-oxopropy diazenyl benzoic acids and studied the spectral correlation. 
Within the above view, there is no reported for the spectral qsar studies of substituted quinoxalines in the past. Therefore the author have taken efforts to prepare some 5- and 6substituted quinoxalines and recorded infrared and ${ }^{13} \mathrm{C}$ NMR spectra for the spectral correlations study.

\section{EXPERIMENTAL}

\section{1. General}

Sigma-Aldrich and Merck company chemicals and solvents used in this present study. The infrared spectra of all chalcones were recorded in SHIMADUZ Fourier Transform IR spectrophotometer using $\mathrm{KBr}$ discs. The ${ }^{13} \mathrm{C}$ NMR spectra of all compounds have been recorded in BRUKER AV 400 type spectrometer, using $\mathrm{CDCl}_{3}$ as a solvent, $100 \mathrm{MHz}$ for ${ }^{13} \mathrm{C}$ NMR spectra, taking TMS as standard.

\section{2. Synthesis of 5- and 6-substituted quinoxalines.}

The 5- and 6-substituted quinoxalines were synthesized and the purities of the compounds were examined by literature method [1,29-31]. The general structure of the 5and 6- substituted quinoxalines is shown in Fig. 1.

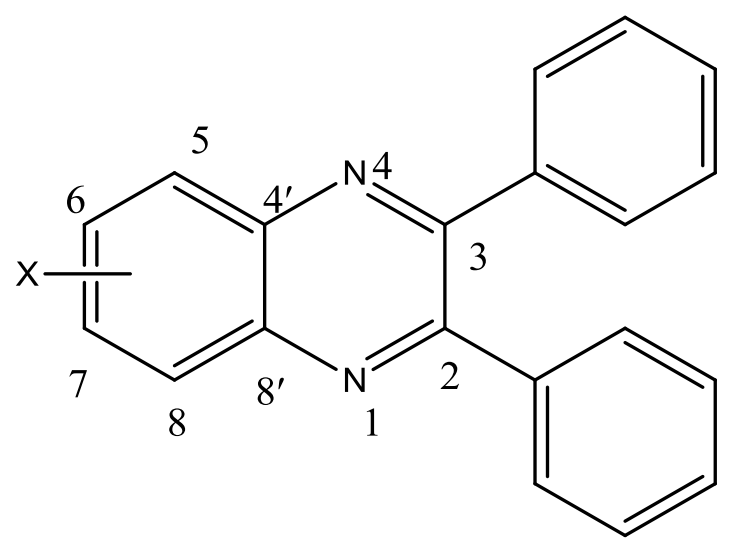

$$
\mathrm{X}=6-\mathrm{H}, 6-\mathrm{PhCO}, 6-\mathrm{Br}, 6-\mathrm{COOH}, 6-\mathrm{Cl}, 6-\mathrm{F}, 6-\mathrm{OCH}_{3}, 5-\mathrm{CH}_{3}, 6-\mathrm{CH}_{3}, 6-\mathrm{NO}_{2}
$$

Fig. 1. General structure of substituted quinoxalines.

\section{RESULTS AND DISCUSSION}

In the present study, the authors have correlated the infrared and ${ }^{13} \mathrm{C}$-NMR spectral frequencies with Hammett substituent constants, $\mathrm{F}$ and $\mathrm{R}$ parameters using single and multilinear regression analysis. Present investigated compounds structure is shown is Fig. 1. It had symmetric structure. The substituents are in 5 and $6^{\text {th }}$ position. With respect to $\mathrm{C}_{2}=\mathrm{N}_{1}$ and $\mathrm{C}_{8}=\mathrm{N}_{1}$, the substituents attached in 5 and $6^{\text {th }}$ positions are considered as meta- and parapositions. Similarly, the $\mathrm{C}_{3}=\mathrm{N}_{4}$ and $\mathrm{C}_{4}=\mathrm{N}_{4}$, substituents attached in 5 and $6^{\text {th }}$ positions are considered as ortho- and meta- positions. Within the considerations, the authors have performed the assigned spectral frequencies were correlated separately by the correlations performed with respect to $\mathrm{C}_{2}=\mathrm{N}_{1}$ and correlations performed with respect to $\mathrm{C}_{4}=\mathrm{N}_{4}$ systems 
in the quinoxalines. The same trend was observed whether the substituents are attached in 8 and $7^{\text {th }}$ positions.

\section{1. Infrared spectral correlation}

The assigned the $\mathrm{C}=\mathrm{N}$ stretches $\left(\mathrm{cm}^{-1}\right)$ of the present investigation substituted quinoxalines were tabulated in Table 1. These data were correlated with Hammett substituent constants, $\mathrm{F}$ and $\mathrm{R}$ parameters [12,14-28]. In this correlation, the Hammett equation was employed as,

Table 1. The infrared $\mathrm{C}=\mathrm{N}$ stretches $\left(\mathrm{cm}^{-1}\right)$ and ${ }^{13} \mathrm{C}$ chemical shifts $(\mathrm{ppm})$ of substituted quinoxalines.

\begin{tabular}{|c|c|c|c|c|c|c|c|}
\hline S1. No. & $\mathrm{X}$ & $v \mathrm{C}=\mathrm{N}$ & $\delta \mathrm{C}_{2}=\mathrm{N}_{1}$ & $\delta \mathrm{C}_{3}=\mathrm{N}_{4}$ & $\delta \mathrm{C}_{4},-\mathrm{N}_{4}$ & $\delta \mathrm{C}_{8^{\prime}-\mathrm{N}_{1}}$ & $\delta \mathrm{C}_{\text {ipso }}$ \\
\hline 1 & $6-\mathrm{H}$ & 1567 & 153.49 & 154.75 & 141.24 & 139.10 & 129.95 \\
\hline 2 & $6-\mathrm{COPh}$ & 1573 & 156.38 & 155.04 & 143.72 & 141.69 & 141.43 \\
\hline 3 & $6-\mathrm{Br}$ & 1569 & 156.47 & 157.82 & 141.44 & 143.97 & 118.32 \\
\hline 4 & $6-\mathrm{COOH}$ & 1563 & 157.64 & 156.64 & 141.73 & 144.11 & 135.60 \\
\hline 5 & $6-\mathrm{Cl}$ & 1568 & 154.23 & 154.31 & 145.16 & 141.74 & 143.77 \\
\hline 6 & $6-\mathrm{F}$ & 1571 & 155.04 & 156.72 & 143.09 & 140.62 & 166.53 \\
\hline 7 & $6-\mathrm{OCH}_{3}$ & 1552 & 153.08 & 156.74 & 144.37 & 139.32 & 162.23 \\
\hline 8 & $5-\mathrm{CH}_{3}$ & 1556 & 153.02 & 155.71 & 143.10 & 141.13 & 140.26 \\
\hline 9 & $6-\mathrm{CH}_{3}$ & 1557 & 153.18 & 156.41 & 143.22 & 141.63 & 139.32 \\
\hline 10 & $6-\mathrm{NO}_{2}$ & 1576 & 160.21 & 164.32 & 142.73 & 145.73 & 142.77 \\
\hline
\end{tabular}

$$
v=\rho \sigma+v_{\mathrm{o}}
$$

where $v_{0}$ is the frequency for the parent member of the series.

The results of statistical analysis are tabulated in Table 2. From Table 2, the single parameter correlation of $\mathrm{vC}=\mathrm{N} m$ - and $p$ - substituted quinoxalines gave satisfactory correlation coefficients with Hammett substituent constants, $\mathrm{F}$ and $\mathrm{R}$ parameters except fluoro substituent for $\sigma_{\mathrm{I}}$ and $\mathrm{R}$ parameter. Similarly the single parameter correlation of these stretches of $o$ - and $m$ - substituted quinoxalines gave satisfactory correlation with Hammett $\sigma$, $\sigma^{+}, \sigma_{I}$ constants and $\mathrm{F}$ parameters. The Hammett $\sigma_{\mathrm{R}}$ constants and $\mathrm{R}$ parameter were failing in correlations. All correlations gave positive $\rho$ values. This meant that the normal substituent effect operates in all systems.

The failure in correlation was due to the inability of predicting the substituent effects on the frequencies along with the resonance conjugative structure as shown in Fig. 2. 


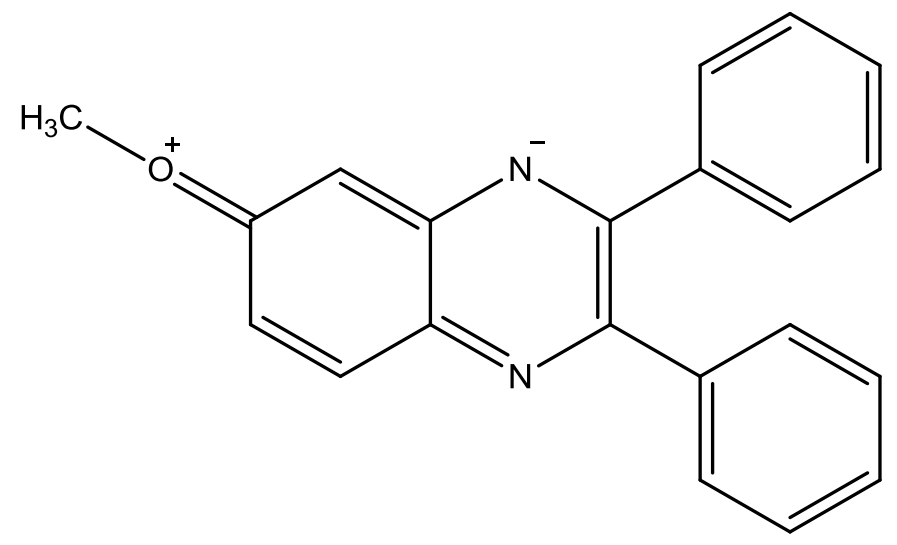

Fig. 2. The resonance-conjugative structure.

Table 2. Results of statistical analysis of IR and ${ }^{13} \mathrm{C}$ NMR spectral values of substituted quinoxalines with Hammett $\sigma, \sigma^{+}, \sigma_{\mathrm{I}}, \sigma_{\mathrm{R}}$ constants, F and R parameters.

\begin{tabular}{|c|c|c|c|c|c|c|c|}
\hline Frequency & $\begin{array}{c}\text { Constan } \\
\mathrm{t}\end{array}$ & $\mathrm{r}$ & I & $\rho$ & S & $\mathrm{n}$ & Correlated derivatives \\
\hline \multicolumn{8}{|c|}{ Correlations performed with $p$-substitution pattern } \\
\hline \multirow{6}{*}{$v \mathrm{C}=\mathrm{N}\left(\mathrm{cm}^{-1}\right)$} & $\sigma$ & 0.917 & 1561.70 & 19.871 & 5.38 & \multirow{6}{*}{10} & \multirow{6}{*}{$\begin{array}{c}\text { 6-H, 6-COPh, 6-Br, } \\
\text { 6-COOH, 6-Cl, 6-F, } \\
\text { 6- }-\mathrm{OCH}_{3}, 5-\mathrm{CH}_{3}, \\
\text { 6- } \mathrm{OCH}_{3}, 6-\mathrm{NO}_{2}\end{array}$} \\
\hline & $\sigma^{+}$ & 0.918 & 1594.41 & 14.100 & 4.15 & & \\
\hline & $\sigma_{\mathrm{I}}$ & 0.907 & 1558.27 & 23.022 & 6.21 & & \\
\hline & $\sigma_{\mathrm{R}}$ & 0.903 & 1567.00 & 16.012 & 8.02 & & \\
\hline & $\mathrm{F}$ & 0.906 & 1558.78 & 19.017 & 6.90 & & \\
\hline & $\mathrm{R}$ & 0.902 & 1566.01 & 7.449 & 8.42 & & \\
\hline \multirow{6}{*}{$\delta \mathrm{C}_{2}=\mathrm{N}_{1}(\mathrm{ppm})$} & $\sigma$ & 0.945 & 154.15 & 6.864 & 0.81 & \multirow{6}{*}{10} & \multirow{6}{*}{$\begin{array}{c}\text { 6- } \mathrm{H}, 6-\mathrm{COPh}, 6-\mathrm{Br}, \\
\text { 6- } \mathrm{COOH}, 6-\mathrm{Cl}, 6-\mathrm{F}, \\
\text { 6- } \mathrm{OCH}_{3}, 5-\mathrm{CH}_{3}, \\
\text { 6- } \mathrm{OCH}_{3}, 6-\mathrm{NO}_{2}\end{array}$} \\
\hline & $\sigma^{+}$ & 0.927 & 155.13 & 4.022 & 0.73 & & \\
\hline & $\sigma_{\mathrm{I}}$ & 0.907 & 153.35 & 6.655 & 1.77 & & \\
\hline & $\sigma_{R}$ & 0.904 & 156.03 & 5.857 & 2.17 & & \\
\hline & $\mathrm{F}$ & 0.912 & 153.35 & 5.930 & 1.87 & & \\
\hline & $\mathrm{R}$ & 0.905 & 156.04 & 4.703 & 2.06 & & \\
\hline \multirow{6}{*}{$\delta \mathrm{C}_{3}=\mathrm{N}_{4}(\mathrm{ppm})$} & $\sigma$ & 0.905 & 156.07 & 4.983 & 2.24 & \multirow{6}{*}{10} & \multirow{6}{*}{$\begin{array}{c}\text { 6-H, 6-COPh, 6-Br, } \\
\text { 6-COOH, 6-Cl, 6-F, } \\
\text { 6- }-\mathrm{OCH}_{3}, 5-\mathrm{CH}_{3}, \\
\text { 6- } \mathrm{OCH}_{3}, 6-\mathrm{NO}_{2}\end{array}$} \\
\hline & $\sigma^{+}$ & 0.904 & 156.80 & 2.372 & 2.27 & & \\
\hline & $\sigma_{\mathrm{I}}$ & 0.905 & 155.18 & 5.862 & 2.55 & & \\
\hline & $\sigma_{R}$ & 0.831 & 157.42 & 4.231 & 2.85 & & \\
\hline & $\mathrm{F}$ & 0.901 & 155.14 & 5.372 & 2.58 & & \\
\hline & $\mathrm{R}$ & 0.825 & 157.03 & 2.513 & 2.89 & & \\
\hline \multirow{6}{*}{$\delta \mathrm{C}_{4^{\prime}}-\mathrm{N}_{4}(\mathrm{ppm})$} & $\sigma$ & 0.802 & 143.14 & -0.849 & 1.31 & \multirow{6}{*}{10} & \multirow{6}{*}{$\begin{array}{c}\text { 6-H, 6-COPh, 6-Br, } \\
\text { 6-COOH, 6-Cl, 6-F, } \\
\text { 6- } \mathrm{OCH}_{3}, 5-\mathrm{CH}_{3}, \\
\text { 6- } \mathrm{OCH}_{3}, 6-\mathrm{NO}_{2}\end{array}$} \\
\hline & $\sigma^{+}$ & 0.836 & 143.04 & -0.912 & 1.24 & & \\
\hline & $\sigma_{\mathrm{I}}$ & 0.890 & 142.84 & 0.547 & 1.33 & & \\
\hline & $\sigma_{\mathrm{R}}$ & 0.830 & 142.76 & -1.890 & 1.28 & & \\
\hline & $\bar{F}$ & 0.810 & 142.94 & 0.173 & 1.34 & & \\
\hline & $\mathrm{R}$ & 0.841 & 142.66 & -2.145 & 1.18 & & \\
\hline$\delta \mathrm{C}_{8^{\prime}}-\mathrm{N}_{1}(\mathrm{ppm})$ & $\sigma$ & 0.980 & 141.11 & 5.190 & 1.34 & 10 & 6-H, 6-COPh, 6-Br, \\
\hline
\end{tabular}




\begin{tabular}{|c|c|c|c|c|c|c|c|}
\hline & $\sigma^{+}$ & 0.969 & 141.85 & 2.907 & 1.58 & & \multirow{5}{*}{$\begin{array}{c}\text { 6- } \mathrm{COOH}, 6-\mathrm{Cl}, 6-\mathrm{F}, \\
\text { 6- } \mathrm{OCH}_{3}, 5-\mathrm{CH}_{3}, \\
\text { 6- } \mathrm{OCH}_{3}, 6-\mathrm{NO}_{2}\end{array}$} \\
\hline & $\sigma_{\mathrm{I}}$ & 0.905 & 140.72 & 4.251 & 1.93 & & \\
\hline & $\sigma_{R}$ & 0.902 & 142.63 & 5.293 & 1.93 & & \\
\hline & $\mathrm{F}$ & 0.884 & 140.75 & 3.733 & 1.97 & & \\
\hline & $\mathrm{R}$ & 0.915 & 142.68 & 4.508 & 1.78 & & \\
\hline \multirow{6}{*}{$\delta C_{\text {ipso }}(p p m)$} & $\sigma$ & 0.827 & 144.02 & $\begin{array}{c}- \\
11.886 \\
\end{array}$ & 14.30 & \multirow{6}{*}{10} & \multirow{6}{*}{$\begin{array}{c}\text { 6- } \mathrm{H}, 6-\mathrm{COPh}, 6-\mathrm{Br} \text {, } \\
\text { 6-COOH, 6-Cl, 6-F, } \\
\text { 6- } \mathrm{OCH}_{3}, 5-\mathrm{CH}_{3}, \\
\text { 6- } \mathrm{OCH}_{3}, 6-\mathrm{NO}_{2}\end{array}$} \\
\hline & $\sigma^{+}$ & 0.841 & 142.53 & $\begin{array}{c}- \\
11.648\end{array}$ & 13.64 & & \\
\hline & $\sigma_{\mathrm{I}}$ & 0.822 & 138.52 & 12.087 & 14.52 & & \\
\hline & $\sigma_{\mathrm{R}}$ & 0.906 & 136.54 & $\begin{array}{c}- \\
44.514\end{array}$ & 11.54 & & \\
\hline & $\mathrm{F}$ & 0.834 & 136.07 & 18.274 & 13.97 & & \\
\hline & $\mathrm{R}$ & 0.904 & 137.11 & $\begin{array}{c}- \\
31.071\end{array}$ & 11.59 & & \\
\hline \multicolumn{8}{|c|}{ Correlations performed with $m$-substitution pattern } \\
\hline \multirow{6}{*}{$v \mathrm{C}=\mathrm{N}\left(\mathrm{cm}^{-1}\right)$} & $\sigma$ & 0.907 & 1554.92 & 22.621 & 5.40 & & \multirow{6}{*}{$\begin{array}{c}\text { 6- } \mathrm{H}, 6-\mathrm{COPh}, 6-\mathrm{Br}, \\
\text { 6-COOH, 6-Cl, 6-F, } \\
\text { 6- } \mathrm{OCH}_{3}, 5-\mathrm{CH}_{3}, \\
\text { 6- } \mathrm{OCH}_{3}, 6-\mathrm{NO}_{2}\end{array}$} \\
\hline & $\sigma^{+}$ & 0.906 & 1562.60 & 10.908 & 6.86 & & \\
\hline & $\sigma_{\mathrm{I}}$ & 0.904 & 1558.54 & 22.871 & 5.92 & 10 & \\
\hline & $\sigma_{\mathrm{R}}$ & 0.881 & 1567.48 & 17.534 & 7.54 & 10 & \\
\hline & $\mathrm{F}$ & 0.901 & 1559.43 & 17.411 & 6.77 & & \\
\hline & $\mathrm{R}$ & 0.826 & 1566.37 & 7.391 & 8.17 & & \\
\hline \multirow{6}{*}{$\delta \mathrm{C}_{2}=\mathrm{N}_{1}(\mathrm{ppm})$} & $\sigma$ & 0.908 & 153.37 & 7.841 & 1.17 & & \multirow{6}{*}{$\begin{array}{c}\text { 6- } \mathrm{H}, 6-\mathrm{COPh}, 6-\mathrm{Br}, \\
\text { 6- } \mathrm{COOH}, 6-\mathrm{Cl}, 6-\mathrm{F}, \\
\text { 6-OCH }, 5-\mathrm{CH}_{3}, \\
\text { 6- } \mathrm{OCH}_{3}, 6-\mathrm{NO}_{2}\end{array}$} \\
\hline & $\sigma^{+}$ & 0.906 & 154.37 & 3.853 & 1.87 & & \\
\hline & $\sigma_{\mathrm{I}}$ & 0.907 & 153.22 & 7.029 & 1.73 & 10 & \\
\hline & $\sigma_{R}$ & 0.905 & 156.07 & 6.139 & 2.17 & 10 & \\
\hline & $\mathrm{F}$ & 0.906 & 153.38 & 5.721 & 7.92 & & \\
\hline & $\mathrm{R}$ & 0.905 & 156.02 & 4.717 & 2.09 & & \\
\hline \multirow{6}{*}{$\delta \mathrm{C}_{3}=\mathrm{N}_{4}(\mathrm{ppm})$} & $\sigma$ & 0.963 & 155.22 & 6.703 & 2.32 & & \multirow{6}{*}{$\begin{array}{c}\text { 6- } \mathrm{H}, 6-\mathrm{COPh}, 6-\mathrm{Br}, \\
\text { 6- } \mathrm{COOH}, 6-\mathrm{Cl}, 6-\mathrm{F}, \\
\text { 6- } \mathrm{OCH}_{3}, 5-\mathrm{CH}_{3}, \\
\text { 6- } \mathrm{OCH}_{3}, 6-\mathrm{NO}_{2}\end{array}$} \\
\hline & $\sigma^{+}$ & 0.904 & 156.19 & 2.741 & 2.75 & & \\
\hline & $\sigma_{\mathrm{I}}$ & 0.905 & 155.12 & 3.299 & 2.51 & 10 & \\
\hline & $\sigma_{R}$ & 0.830 & 157.41 & 4.370 & 2.86 & 10 & \\
\hline & $\mathrm{F}$ & 0.905 & 153.45 & 6.357 & 2.58 & & \\
\hline & $\mathrm{R}$ & 0.825 & 155.07 & 2.532 & 2.91 & & \\
\hline \multirow{6}{*}{$\delta \mathrm{C}_{4^{\prime}}-\mathrm{N}_{4}(\mathrm{ppm})$} & $\sigma$ & 0.800 & 142.98 & -0.033 & 1.33 & & \multirow{6}{*}{$\begin{array}{c}\text { 6-H, 6-COPh, 6-Br, } \\
\text { 6- } \mathrm{COOH}, 6-\mathrm{Cl}, 6-\mathrm{F} \text {, } \\
\text { 6- } \mathrm{OCH}_{3}, 5-\mathrm{CH}_{3}, \\
\text { 6- } \mathrm{OCH}_{3}, 6-\mathrm{NO}_{2}\end{array}$} \\
\hline & $\sigma^{+}$ & 0.808 & 142.91 & 0.268 & 1.33 & & \\
\hline & $\sigma_{I}$ & 0.812 & 142.79 & 0.678 & 1.32 & & \\
\hline & $\sigma_{R}$ & 0.813 & 142.72 & -1.927 & 1.27 & 10 & \\
\hline & $\mathrm{F}$ & 0.806 & 142.88 & 0.295 & 1.33 & & \\
\hline & $\mathrm{R}$ & 0.847 & 142.64 & -2.138 & 1.17 & & \\
\hline \multirow{6}{*}{$\delta \mathrm{C}_{8^{\prime}}-\mathrm{N}_{1}(\mathrm{ppm})$} & $\sigma$ & 0.970 & 140.53 & 5.641 & 1.57 & \multirow{6}{*}{10} & \multirow{6}{*}{$\begin{array}{c}\text { 6-H, 6-COPh, 6-Br, } \\
\text { 6- } \mathrm{COOH}, 6-\mathrm{Cl}, 6-\mathrm{F}, \\
\text { 6-OCH }, 5-\mathrm{CH}_{3}, \\
\text { 6- } \mathrm{OCH}_{3}, 6-\mathrm{NO}_{2}\end{array}$} \\
\hline & $\sigma^{+}$ & 0.908 & 141.43 & 1.968 & 2.08 & & \\
\hline & $\sigma_{\mathrm{I}}$ & 0.905 & 140.48 & 4.947 & 1.84 & & \\
\hline & $\sigma_{R}$ & 0.900 & 142.59 & 5.343 & 1.94 & & \\
\hline & $\mathrm{F}$ & 0.848 & 140.64 & 3.791 & 1.97 & & \\
\hline & $\mathrm{R}$ & 0.906 & 142.64 & 4.528 & 1.80 & & \\
\hline$\delta \mathrm{C}_{\mathrm{ipso}}(\mathrm{ppm})$ & $\sigma$ & 0.817 & 141.79 & 0.925 & 14.88 & 10 & 6-H, 6-COPh, 6-Br, \\
\hline
\end{tabular}




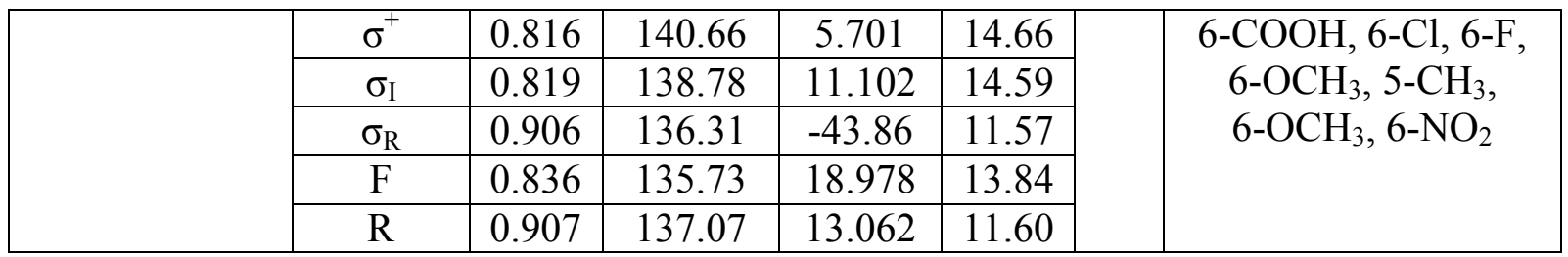

$\mathrm{r}=$ correlation coefficient; $\mathrm{I}=$ intercept; $\rho=$ slope $\mathrm{s}=$ standard deviation; $\mathrm{n}=$ number of correlated derivatives

\section{2. ${ }^{13} \mathrm{C}$ NMR spectral correlation}

The assigned the ${ }^{13} \mathrm{C}$ NMR chemical shifts of substituted quinoxalines are presented in Table 1. These data are correlated with Hammett substituent constants, $\mathrm{F}$ and $\mathrm{R}$ parameters using single and multi-regression analysis [12,14-28]. In these correlations, the Hammett equation was taken in the form as,

$$
\delta=\delta_{0}+\rho \sigma
$$

where $\delta_{0}$ is the chemical shift of the corresponding parent compound.

The results of statistical analyses are shown in Table 2. The correlations performed with respect to $\mathrm{C}_{2}=\mathrm{N}_{1}$ ( $p$-substitution pattern), chemical shifts of $\delta \mathrm{C}_{2}=\mathrm{N}_{1}$ (ppm) gave satisfactory correlation with Hammett substituent constants, $\mathrm{F}$ and $\mathrm{R}$ parameters. Hammett $\sigma_{\mathrm{R}}$ and $\mathrm{F}$ parameter gave satisfactory correlation except fluoro substituent. The $\delta \mathrm{C}_{3}=\mathrm{N}_{4}(\mathrm{ppm})$ chemical shifts with Hammett $\sigma$ and $\sigma^{+}$constants gave satisfactory correlations except nitro substituent. The satisfactory correlations observed for Hammett $\sigma_{\mathrm{I}}$ and $\mathrm{F}$ parameters. The Hammett $\sigma_{R}$ constant and $\mathrm{R}$ parameters were fails in correlations. All correlations gave positive $\rho$ values. These mean that the normal substituent effect operates in all systems. The reason for the failure in the correlation was already stated and along with the resonance conjugative structure as shown in Fig. 2.

The single parameter correlation of $\delta \mathrm{C}_{4},-\mathrm{N}_{4}(\mathrm{ppm})$ chemical shifts with Hammett substituent constants, $\mathrm{F}$ and $\mathrm{R}$ were failed. In these correlations, some of the negative $\rho$ values obtained. This negative $\rho$ values reduced correlations considerably. The reasons for this poor correlations were already stated and along with the resonance conjugative structure as shown in Fig. 2.

The single parameter correlation of $\delta \mathrm{C}_{8},-\mathrm{N}_{1}(\mathrm{ppm})$ chemical shifts with Hammett substituent constants, $\mathrm{F}$ and $\mathrm{R}$ gave satisfactory $\mathrm{r}$ values. The $\mathrm{F}$ parameter was failed in the correlation. All correlations gave positive $\rho$ values. The reason for the poor correlation of $F$ parameter was already stated and along with the resonance conjugative structure as shown in Fig. 2.

The ipso carbon chemical shifts of the quinoxalines with Hammett $\sigma_{\mathrm{R}}$ constant and $\mathrm{R}$ parameters gave satisfactory correlations. The Hammett $\sigma, \sigma^{+}, \sigma_{\mathrm{I}}$ constants and $\mathrm{F}$ parameter were fail in correlations. The inability of substituents along with conjugative structure as shown in Fig. 2. is explain the reasons for poor correlations.

The correlations performed with respect to $\mathrm{C}_{4}=\mathrm{N}_{3}$ ( $m$ - substitution pattern), the chemical shifts of $\delta \mathrm{C}_{2}=\mathrm{N}_{1}(\mathrm{ppm})$ gave satisfactory correlation with Hammett substituent constants, $\mathrm{F}$ and R parameters. The $\delta \mathrm{C}_{3}=\mathrm{N}_{4}(\mathrm{ppm})$ chemical shifts with Hammett $\sigma, \sigma^{+}$and $\sigma_{\mathrm{I}}$ constants and $\mathrm{F}$ parameter constants gave satisfactory correlations. The Hammett $\sigma_{\mathrm{R}}$ constant and R parameters were failed in correlations. All correlations gave positive $\rho$ values. These mean that the normal substituent effect operates in all systems. The reason for the failure in 
the correlation was already stated and along with the resonance conjugative structure as shown in Fig. 2.

The chemical shifts $\delta \mathrm{C}_{4}-\mathrm{N}_{4}$ (ppm) of quinoxalines with Hammett substituent constants, $\mathrm{F}$ and $\mathrm{R}$ gave poor correlation. Some of correlations gave the negative $\rho$ values obtained in this correlation. This negative $\rho$ values reduced correlations considerably. The reasons for this poor correlations were already stated and along with the resonance conjugative structure as shown in Fig. 2.

The single parameter correlation of $\delta \mathrm{C}_{8},-\mathrm{N}_{1}(\mathrm{ppm})$ chemical shifts with Hammett substituent constants and $\mathrm{F}$ gave satisfactory $\mathrm{r}$ values. The $\mathrm{R}$ parameter was failed in the correlation. All correlations gave positive $\rho$ values. The reason for the poor correlation of $\mathrm{R}$ parameter was already stated and along with the resonance conjugative structure shown in Fig. 2.

The ipso carbon chemical shifts of the quinoxalines with Hammett $\sigma_{\mathrm{I}}$ constant and $\mathrm{R}$ parameters gave satisfactory correlations. The Hammett $\sigma, \sigma^{+}, \sigma_{\mathrm{R}}$ constants and F parameters were failed in correlations. Already stated the reason for the poor correlations and it is along with conjugative structure as shown in Fig. 2.

Some of the single parameters were failed in correlations with Hammett substituent constants, $\mathrm{F}$ and $\mathrm{R}$ parameters. While seeking multi-linear correlations with $\sigma_{\mathrm{I}}$ and $\sigma_{\mathrm{R}}$ constants or Swain Lupton's [32] F and R parameters gave satisfactory correlations for infrared and ${ }^{13} \mathrm{C}$ NMR spectral data of quinoxalines. The generated multi-regression analysis equations are shown in (3-26)

Correlations performed with $p$-substitution pattern

$$
\begin{aligned}
& \nu \mathrm{C}=\mathrm{N}\left(\mathrm{cm}^{-1}\right)=1560.21( \pm 2.659)+24.624( \pm 6.737) \sigma_{\mathrm{I}}+19.201( \pm 8.394) \sigma_{\mathrm{R}} \\
& (R=0.984, \mathrm{n}=10, \mathrm{P}>95 \%) \\
& v \mathrm{C}=\mathrm{N}\left(\mathrm{cm}^{-1}\right)=1559.93( \pm 3.652)+20.044( \pm 8.589) \mathrm{F}+9.360( \pm 0.817) \mathrm{R} \\
& (R=0.968, \mathrm{n}=10, \mathrm{P}>95 \%) \\
& \delta \mathrm{C}_{2}=\mathrm{N}_{1}(\mathrm{ppm})=154.03( \pm 0.588)+7.222( \pm 1.490) \sigma_{\mathrm{I}}+6.739( \pm 1.857) \sigma_{\mathrm{R}} \\
& (R=0.990, \mathrm{n}=10, \mathrm{P}>95 \%) \\
& \delta \mathrm{C}_{2}=\mathrm{N}_{1}(\mathrm{ppm})=154.01( \pm 0.578)+6.514( \pm 1.360) \mathrm{F}+5.324( \pm 1.268) \mathrm{R} \\
& (R=0.991, \mathrm{n}=10, \mathrm{P}>95 \%) \\
& \delta \mathrm{C}_{3}=\mathrm{N}_{4}(\mathrm{ppm})=155.69( \pm 1.310)+6.283( \pm 3.319) \sigma_{\mathrm{I}}+5.045( \pm 2.142) \sigma_{\mathrm{R}} \\
& (R=0.963, \mathrm{n}=10, \mathrm{P}>95 \%) \\
& \delta \mathrm{C}_{3}=\mathrm{N}_{4}(\mathrm{ppm})=155.51( \pm 1.397)+5.710( \pm 3.285) \mathrm{F}+3.063( \pm 0.306) \mathrm{R} \\
& (R=0.958, \mathrm{n}=10, \mathrm{P}>90 \%) \\
& \delta \mathrm{C}_{4},=\mathrm{N}_{4}(\mathrm{ppm})=142.66( \pm 0.722)+0.394( \pm 0.183) \sigma_{\mathrm{I}}-1.838( \pm 0.221) \sigma_{\mathrm{R}} \\
& (R=0.930, \mathrm{n}=10, \mathrm{P}>90 \%) \\
& \delta \mathrm{C}_{4}=\mathrm{N}_{4}(\mathrm{ppm})=142.68( \pm 0.682)-0.062( \pm 0.006) \mathrm{F}-2.152( \pm 0.127) \mathrm{R} \\
& (R=0.947, \mathrm{n}=10, \mathrm{P}>90 \%)
\end{aligned}
$$




$$
\begin{gathered}
\delta \mathrm{C}_{8^{\prime}}=\mathrm{N}_{1}(\mathrm{ppm})=141.324( \pm 0.831)+4.750( \pm 2.107) \sigma_{\mathrm{I}}+5.907( \pm 2.623) \sigma_{\mathrm{R}} \\
(R=0.975, \mathrm{n}=10, \mathrm{P}>95 \%) \\
\delta \mathrm{C}_{8^{\prime}}=\mathrm{N}_{1}(\mathrm{ppm})=141.35( \pm 0.768)+4.272( \pm 1.801) \mathrm{F}+4.915( \pm 1.685) \mathrm{R} \\
(R=0.980, \mathrm{n}=10, \mathrm{P}>95 \%) \\
\delta \mathrm{C}_{\mathrm{ipso}}(\mathrm{ppm})=134.19( \pm 6.400)+8.501( \pm 1.621) \sigma_{\mathrm{I}}-42.971( \pm 20.204) \sigma_{\mathrm{R}} \\
(R=0.964, \mathrm{n}=10, \mathrm{P}>95 \%) \\
\delta \mathrm{C}_{\mathrm{ipso}}(\mathrm{ppm})=133.42( \pm 6.258)+15.023( \pm 1.472) \mathrm{F}-29.343( \pm 13.708) \mathrm{R} \\
(R=0.968, \mathrm{n}=10, \mathrm{P}>95 \%)
\end{gathered}
$$

Correlations performed with $m$-substitution pattern

$$
\begin{gathered}
\nu \mathrm{C}=\mathrm{N}\left(\mathrm{cm}^{-1}\right)=1560.79( \pm 2.527)+23.558( \pm 6.304) \sigma_{\mathrm{I}}+18.850( \pm 7.737) \sigma_{\mathrm{R}} \\
(R=0.985, \mathrm{n}=10, \mathrm{P}>95 \%)
\end{gathered}
$$

$v \mathrm{C}=\mathrm{N}\left(\mathrm{cm}^{-1}\right)=1549.43( \pm 3.496)+17.414( \pm 8.345) \mathrm{F}+14.321( \pm 6.173) \mathrm{R}$

$$
(R=0.968, \mathrm{n}=10, \mathrm{P}>95 \%)
$$

$$
\begin{gathered}
\delta \mathrm{C}_{2}=\mathrm{N}_{1}(\mathrm{ppm})=154.00( \pm 0.603)+7.264( \pm 1.505) \sigma_{\mathrm{I}}+6.532( \pm 1.847) \sigma_{\mathrm{R}} \\
(R=0.991, \mathrm{n}=10, \mathrm{P}>95 \%)
\end{gathered}
$$

$$
\delta \mathrm{C}_{2}=\mathrm{N}_{1}(\mathrm{ppm})=154.01( \pm 0.582)+6.437( \pm 1.354) \mathrm{F}+5.482( \pm 1.297) \mathrm{R}
$$$$
(R=0.991, \mathrm{n}=10, \mathrm{P}>95 \%)
$$

$$
\delta \mathrm{C}_{3}=\mathrm{N}_{4}(\mathrm{ppm})=155.01( \pm 1.270)+6.229( \pm 3.400) \sigma_{\mathrm{I}}+4.720( \pm 0.407) \sigma_{\mathrm{R}}
$$

$$
(R=0.964, \mathrm{n}=10, \mathrm{P}>95 \%)
$$

$$
\delta \mathrm{C}_{3}=\mathrm{N}_{4}(\mathrm{ppm})=155.44( \pm 1.372)+5.766( \pm 3.189) \mathrm{F}+3.223( \pm 0.132) \mathrm{R}
$$

$$
(R=0.960, \mathrm{n}=10, \mathrm{P}>90 \%)
$$

$$
\begin{gathered}
\delta \mathrm{C}_{4}=\mathrm{N}_{4}(\mathrm{ppm})=142.57( \pm 0.733)+0.563( \pm 0.183) \sigma_{\mathrm{I}}-1.894( \pm 0.224) \sigma_{\mathrm{R}} \\
(R=0.932, \mathrm{n}=10, \mathrm{P}>90 \%)
\end{gathered}
$$

$$
\begin{gathered}
\delta \mathrm{C}_{4^{\prime}}=\mathrm{N}_{4}(\mathrm{ppm})=142.63( \pm 0.671)+0.016( \pm 0.005) \mathrm{F}-2.136( \pm 0.149) \mathrm{R} \\
(R=0.947, \mathrm{n}=10, \mathrm{P}>90 \%)
\end{gathered}
$$

$$
\delta \mathrm{C}_{8}=\mathrm{N}_{1}(\mathrm{ppm})=141.13( \pm 0.815)+5.149( \pm 2.033) \sigma_{\mathrm{I}}+5.622( \pm 0.246) \sigma_{\mathrm{R}}
$$$$
(R=0.978, \mathrm{n}=10, \mathrm{P}>95 \%)
$$

$$
\begin{gathered}
\delta \mathrm{C}_{8^{\prime}}=\mathrm{N}_{1}(\mathrm{ppm})=141.23( \pm 0.725)+4.453( \pm 1.696) \mathrm{F}+5.061( \pm 1.624) \mathrm{R} \\
(R=0.982, \mathrm{n}=10, \mathrm{P}>95 \%)
\end{gathered}
$$

$$
\delta \mathrm{C}_{\mathrm{ipso}}(\mathrm{ppm})=133.60( \pm 6.550)+9.543( \pm 1.634) \sigma_{\mathrm{I}}-43.352( \pm 2.062) \sigma_{\mathrm{R}}
$$$$
(R=0.964, \mathrm{n}=10, \mathrm{P}>95 \%)
$$

$$
\begin{gathered}
\delta \mathrm{C}_{\mathrm{ipso}}(\mathrm{ppm})=132.95( \pm 6.148)+15.156( \pm 1.429) \mathrm{F}-29.249( \pm 13.623) \mathrm{R} \\
(R=0.968, \mathrm{n}=10, \mathrm{P}>95 \%)
\end{gathered}
$$




\section{CONCLUSIONS}

A series containing ten substituted 5- and 6- substituted quinoxaline derivatives have been synthesized and examined their purities by literature method. The infrared and ${ }^{13} \mathrm{C}$ NMR spectral frequencies of $\mathrm{C}=\mathrm{N}, \mathrm{C}-\mathrm{N}$ and ipso carbons of the quinoxalines were assigned and correlated based on $m$ - and $p$ - substituted system with Hammett substituent constants, $\mathrm{F}$ and $\mathrm{R}$ parameters using single and multi-regression analysis. From the results of statistical analyses, most of the single parameter correlations and all multi- correlations gave satisfactory correlation coefficients.

\section{References}

[1] A. Hasaninejad, A. Zare, M. R. Mohammadizadeh, M. Shekouhy, Green Chem. Lett. Rev. 3 (2010) 143-148.

[2] A. R. Bendale, D. Kotak, D. P. Damahe, S. P. Narkhede, A. G. Jadhav and G. Vidyasagar, Der Chemica Sinica, 2(2011) 20-24.

[3] M. M. Badran, A. A. Moneer, H. M. Refaat and A. A El-Malah, J. Chin. Chem. Soc. 54 (2007) 469-478.

[4] W. He, M. R. Meyers, B. Hanney, A. Spada, G. Blider, H. Galzeinski, D. Amin, S. Needle, K. Page, Z. Jayyosi and H. Perrone, Bioorg. Med. Chem. Lett. 13(2003) 30973100 .

[5] K. Waisser, Z. Odlerova, R. Beckert, R. Mayer, Pharmazie. 44(1989) 234-235.

[6] L. E. Seitz, W. J. Suling and R. C. Reynolds, J. Med. Chem. 45(2002) 5604-5606.

[7] G. Sakata, K. Makino and Y. Karasawa, Heterocycles, 27(1988) 2481-2515.

[8] R. Sarges, H. R. Howard, R. C. Browne, L. A. Label and P. A. Seymour, J. Med. Chem. 33(1990)2240-2254.

[9] S. T. Hazeldine, L. Polin, J. Kushner, J. Paluch, K. White, M. Edelstein, E. Palomino, T. H. Corbett and J. P. Horwitz, J. Med. Chem. 44(2001) 1758-1776.

[10] S. T. Hazeldine, L. Polin, J. Kushner, K. White, N. M. Bouregeois, B. Crantz, E. Palomino, T. H. Corbett and J. P. Horwitz, J. Med. Chem. 45(2002) 3130-3137.

[11] H. Xu, W. M. Liao, H. F. Li, Ultrason. Sonochem. 14 (2007) 779-782.

[12] K. Sathiyamoorthy, V. Mala, S.P. Sakthinathan, D. Kamalakkannan, R. Suresh, G. Vanangamudi, G. Thirunarayanan, Spectrochim. Acta 112A(2013) 245-256.

[13] S. Sasikala, K. Thirumurthy, P. Mayavel, G. Thirunarayanan, Org. Med. Chem. Lett. (2012.) doi:10.1186/2191-2858-2-20.

[14] R. Suresh, D. Kamalakkannan, K. Ranganathan, R. Arulkumaran, R. Sundararajan, S.P. Sakthinathan, S. Vijayakumar, K. Sathiyamoorthy, V. Mala, G. Vanangamudi, K. Thirumurthy, P. Mayavel, G. Thirunarayanan, Spectrochim. Acta. 101A (2013) 239248.

[15] G. Thirunarayanan, K. G. Sekar, International Letters of Chemistry, Physics and Astronomy 6 (2014) 39-47. 
[16] R. Arulkumaran, R. Sundararajan, V. Manikandan, V. Sathiyendiran, S. Pazhamalai, G. Thirunarayanan, International Letters of Chemistry, Physics and Astronomy 19(1) (2014) 25-15.

[17] K. G. Sekar, G. Thirunarayanan, International Letters of Chemistry, Physics and Astronomy 8(2) (2013) 160-174.

[18] G. Thirunarayanan, Int. J. Sci. Res. Know. 1(12) (2013) 480-596.

[19] G. Thirunarayanan, G. Vanangamudi, Spectrochim Acta, 81A (2011) 390-396.

[20] K. G Sekar, G. Thirunarayanan, International Letters of Chemistry, Physics and Astronomy 8(3) (2013) 249-258.

[21] G. Thirunarayanan, International Letters of Chemistry, Physics and Astronomy 9(2) (2013) 152-161.

[22] G. Thirunarayanan, M. Suresh, International Letters of Chemistry, Physics and Astronomy 4 (1) (2014) 1-11.

[23] G. Thirunarayanan, Bull. Chem. Soc. Ethiop. 28(1) (2014)73-79.

[24] G. Thirunarayanan, International Letters of Chemistry, Physics and Astronomy 5 (2014) 89-98.

[25] G. Thirunarayanan, K. G. Sekar, J. Taibah Univ. Sci. 8 (2014)124-136.

[26] G. Thirunarayanan, K.G. Sekar, R. Lakshmi Narayanan, International Letters of Chemistry, Physics and Astronomy 13(1) (2014) 88-94.

[27] P. Mayavel, K. Thirumurthy, S. Dineshkumar, G. Thirunarayanan, Q-Science Connect. (2014) DOI: http://dx.doi.org/10.5339/connect.2014.10

[28] V. Sathiyendiran, K. G. Sekar, G. Thirunarayanan, R. Arulkumaran, R. Sundararajan, D. Kamalakkannan, R. Suresh, V. Manikanadan, R. Vijayakumar, G. Vanangamudi, International Letters of Chemistry, Physics and Astronomy 11(1) (2014) 33-43.

[29] F. Hakimi, B. B. F. Mirjalili, Curr. Chem. Lett. 2 (2013) 105-108.

[30] D. M. Ruiz, J. C. Autino, N. Q. Patricia, G. V'azquez, G. P. Romanelli, The Scientific World Journal., Volume 2012, Article ID 174784, 8 pages, doi:10.1100/2012/174784

[31] J. J. Morales-Castellanos, K. Ramírez-Hernández, N. S. Gómez-Flores, O. R. RodasSuárez, J. Peralta-Cruz, Molecules 17 (2012) 5164-5176.

[32] C. G. Swain and E. C. Lupton Jr., J. Am. Chem. Soc. 90 (1968) 4328-4337. 